\title{
CUENTO, TRADUCCIÓN Y TRANSFERENCIAS CULTURALES EN LA REVISTA COLOMBIANA ILUSTRADA EL GRÁFICO (1925-1941)
}

\author{
SHORT STORY, TRANSLATION AND CULTURAL TRANSFERENCES AT THE COLOMBIAN \\ ILLUSTRATED MAGAZINE EL GRÁFICO (1925-1941) \\ CONTE, TRADUCTION ET TRANSFERTS CULTURELS DANS LE MAGAZINE COLOMBIEN \\ ILLUSTRÉ EL GRÁFICO (1925-1941)
}

Paula Andrea Marín Colorado

Directora de la línea de

investigación: "El libro en

Colombia", Instituto Caro y Cuervo,

Bogotá, Colombia. Miembro del

Grupo de Investigación Colombia:

tradiciones de la palabra,

Universidad de Antioquia, Medellín,

Antioquia, Colombia.

Calle 10 N. ${ }^{\circ}$ 4-69, Bogotá, D. C.,

Colombia.

paulanmc@gmail.com
Este artículo se deriva de la investigación "El cuento colombiano en las revistas literarias colombianas (1900-1950). Estudio histórico y hemerográfico", diseñado y ejecutado por el Grupo de Investigación Colombia: tradiciones de la palabra (Universidad de Antioquia, Colombia). Inicio: febrero de 2016. Proyecto inscrito en el marco de la Estrategia de Sostenibilidad para grupos de investigación CODI 2016-2017 (Universidad de Antioquia, Medellín, Colombia).

\begin{abstract}
RESUMEN
El Gráfico. Semanario Ilustrado (1910-1941), fundado por los tipógrafos Alberto Sánchez y Abraham Cortés, nació como una revista para brindar ilustraciones, historia, información, literatura y variedades a sus lectores. En la segunda etapa de la revista (1925-1941), esta tuvo cambios que afectaron la manera en la que el género cuento se venía publicando en sus páginas, tanto el de autoría de escritores colombianos como el de extranjeros. Mediante un análisis de las relaciones entre prensa y literatura, y desde la perspectiva de las transferencias culturales y de los estudios sobre traducción, se muestra la manera en la que El Gráfico se transformó en un medio de comunicación que reafirmó la diferencia entre los circuitos especializados y comerciales de lo literario, y permitió ampliar el repertorio para el cuento colombiano (a partir de sus relaciones con los sistemas literarios francés, ruso y español).
\end{abstract}

Palabras clave: cuento colombiano del siglo $\mathrm{xx}$, transferencias culturales, prensa colombiana del siglo xx, traducción literaria, repertorio literario, El Gráfico. Semanario Ilustrado

\begin{abstract}
El Gráfico. Semanario Ilustrado (1910-1941), founded by typographers Alberto Sánchez and Abraham Cortés, was born as a magazine to provide illustrations, history, information, literature and varieties to its readers. In the second phase of the magazine (1925-1941), the journal had several changes that affected the way in which the short story literary genre was published in its pages, both written by foreign and Colombian correspondents. This paper will show the way in which $E l$ Gráfico became a mass media that reaffirmed the difference between the specialized and commercial circuits of the literary, and allowed to extend the repertoire for the Colombian short story. These processes occurred due to the relations with the French, Russian and Spanish literary system. The analysis proposed in the current study will be based on the relations between the press and literature, on the perspective of cultural transfers and on the translation studies.
\end{abstract}

Keywords: Colombian short story of the twentieth century, cultural transfers, translation, Colombian press of the twentieth century, literary repertoire, El Gráfico. Semanario Ilustrado 


\section{RÉSUMÉ}

El Gráfico. Semanario Ilustrado (1910-1941), fondée par les typographes Alberto Sánchez et Abraham Cortés a d'abord offert à ses lecteurs des illustrations, de l'histoire, des informations, de la littérature et des variétés. Au cours de sa deuxième étape (1925-1941), certains changements ont modifié la façon dont le conte en tant que genre y apparaissait jusque-là, et cela touchait aussi bien les auteurs colombiens que les auteurs étrangers. L'analyse des relations entre la presse et la littérature, et cela du point de vue des transferts culturels et des études sur traduction, montre comment au sein du monde de la presse, El Gráfico a marqué la différence existant entre la presse spécialisée et la presse grand public. Ceci lui a permis d'offrir un répertoire plus large du conte colombien (et en partie grâce à ses relations avec les systèmes littéraires français, russe et espagnol).

Mots-clés : conte colombien $\mathrm{du} \mathrm{Xx}^{\mathrm{e}}$ siècle, transferts culturels, traduction, presse colombienne du $\mathrm{xx}^{\mathrm{e}}$ siècle, répertoire littéraire, El Gráfico. Semanario Ilustrado 


\section{Introducción}

En este trabajo se analiza la revista El Gráfico. Semanario Ilustrado (Bogotá, 1910-1941) como matriz mediática del "artículo-cuento" (Botrel, s. f.) escrito por autores extranjeros, y se caracteriza su poética desde la perspectiva de las transferencias culturales y de los estudios sobre la traducción.

A partir de una investigación anterior en la que se analizó la primera etapa de publicación de $E l$ Gráfico (1910-1924) (Marín Colorado, 2016), este artículo se enfoca en la segunda etapa: 1925-1941.

El Gráfico, a diferencia de otras publicaciones periódicas colombianas de la primera mitad del siglo Xx en donde el cuento ocupa un lugar minoritario, fue la primera revista que incluyó secciones exclusivas para la divulgación de cuentos (en su gran mayoría, de autores colombianos); estas se mantuvieron desde su fundación hasta su cierre. ${ }^{1}$ En su primera etapa, estas secciones se denominaron "Cuentos Nacionales" y "Cuentos Extranjeros"; en la segunda (a partir de la década de los treinta), desaparece la sección "Cuentos Nacionales" y aparece "El Cuento". En esta etapa, los cuentos nacionales impresos son escasos y estos solo vuelven a tener una presencia visible en 1939, cuando se presentan bajo el subtítulo "Cuento Nacional".

En la segunda etapa de la revista, la visión comercial se impone sobre la intelectual y la función del cuento cambia. Sobre todo, a partir de la década de los treinta, los cuentos aparecen con un propósito muy claro: entretener al lector. Si bien en la primera etapa el entretenimiento no era una

1 Además de estas secciones, El Gráfico mantuvo, desde 1925, el suplemento Pulgarcito, dedicado al público infantil; una de sus secciones estaba dedicada al "cuento para niños”. En los últimos dos años de la revista, el suplemento pasó a llamarse "Sección Infantil".

2 La clasificación del corpus de estudio como "cuentos", usada para la escritura de este artículo, proviene, pues, de la denominación utilizada por la revista en estas secciones. función contraria al cuento, no era la más evidente. Los directores de El Gráfico afirmaron, una y otra vez, que su intención era: "Servir a la élite de los poetas y prosadores colombianos de auténtico medio de comunicación con el público de buen gusto" ("Charla con los lectores", 1925, s. d.). Es esta la finalidad que se transforma y cuyas consecuencias se amplían a continuación en este artículo, analizando la manera en la que los cuentos extranjeros aparecieron en la revista, pasando de las "versiones" a las "traducciones", es decir, de una modalidad de traducción especializada a una generalista (Zapata, 2015), en la que el autor de la traducción deja de ser mencionado.

Con los dos apartados siguientes pretendo, pues, demostrar que El Gráfico le dio paulatinamente cabida a la circulación de saberes y discursos cercanos a la cotidianidad y a la actualidad de la mayoría del público lector disponible en la época. Esta circulación se dio dentro de un ámbito menos especializado que el del tradicional circuito letrado, cuyas publicaciones privilegiaron la crítica literaria y la poesía, así como la explicitación de las fuentes de sus traducciones e incluso el contraste entre diferentes versiones del mismo texto (Bedoya Sánchez, 2012). El Gráfico demuestra que, en este período (1925-1941), coexisten ya dos circuitos bien diferenciados de circulación de lo literario: uno especializado, que sirve como mediación del prestigio literario, y otro de tendencia más comercial, útil como mediación de la literatura en tanto producto económico. En este último circuito, el cuento tuvo un lugar privilegiado, pese a que su papel en la trayectoria de consagración del escritor colombiano haya sido marginal.

El análisis de la muestra recopilada para esta investigación de los diecisiete años que configuran la segunda etapa de El Gráfico permitió construir un corpus, que se resume en la tabla 1. Así, al menos 147 (50\%) de los 294 cuentos publicados provinieron de autores extranjeros. Las conclusiones del análisis son examinadas en los siguientes dos apartados. 
Tabla 1 Número de cuentos según lugar de nacimiento de los autores

\begin{tabular}{lcc}
\hline $\begin{array}{l}\text { Lugar de nacimiento } \\
\text { de los autores }\end{array}$ & $\begin{array}{c}\text { Cantidad de } \\
\text { cuentos }\end{array}$ & $\begin{array}{c}\text { Porcentaje } \\
(\%)\end{array}$ \\
\hline Colombia & 115 & 39,11 \\
Francia & 38 & 12,92 \\
España & 12 & 4,08 \\
Rusia & 12 & 4,08 \\
Reino Unido & 10 & 3,4 \\
Argentina & 10 & 3,4 \\
Italia & 8 & 2,72 \\
Uruguay & 7 & 2,38 \\
Estados Unidos & 5 & 1,7 \\
México & 5 & 1,7 \\
Otros países & 40 & 13,6 \\
Sin identificar & 32 & 10,88 \\
Total & 294 & 99,97 \\
\hline
\end{tabular}

* Los otros países son: Austria, Bélgica, Bolivia, Brasil, Chile, Ecuador, Grecia, Hungría, India, Noruega, Perú, Polonia, Portugal, Puerto Rico, República Dominicana, Suiza, Ucrania

Fuente: Datos consolidados por la autora de acuerdo con la matriz de análisis (base de datos) de la investigación, elaborada por el Grupo de Investigación Colombia: tradiciones de la palabra.

Entre los 147 cuentos extranjeros impresos en $E l$ Gráfico en su segunda etapa, se destacan ocho autores (con 30 cuentos en total), correspondientes a los países de donde más provienen los cuentos publicados en la revista: ${ }^{3}$ de Francia, Frederic Boutet (4), Michel Corday —seudónimo de Louis-Édouard Pollet- (4), Camille Mauclair —seudónimo de Séverin Faust- (3), Henri Barbusse (3) y Pierre Nezelof (2); de Rusia, Arkadi Averchenko (6) y Anton Chéjov (5); y de España, Ramón Gómez de la Serna (3).

3 Es evidente que el lugar de nacimiento de los autores es un criterio parcial de investigación, en el que no se tienen en cuenta factores como el lugar de producción, publicación y difusión del autor, u otros más complejos, aun como su identificación cultural o legal con un país específico; haciendo esta salvedad, paso a presentar mi análisis, que pretende mostrar, entonces, un primer avance de las cuestiones aquí planteadas.

Con la selección de estos treinta cuentos, escritos por los autores mencionados (todos más o menos contemporáneos), me referiré a un fenómeno particular, homólogo al resultado de estas estadísticas y que permitirá comprenderlo mejor: la manera en la que las literaturas francesa, rusa y española figuran en la publicación periódica (en el caso de los cuentos franceses y rusos, a partir de la traducción) y su funcionamiento como referentes para los autores colombianos, específicamente sus implicaciones para el género cuento.

Las preguntas para el caso que nos compete aquí son, en primer lugar, en qué medida los cuentos extranjeros divulgados en El Gráfico fueron traducidos en Colombia o fueron recibidos ya como traducciones; y, en segundo lugar, en qué grado esas traducciones pudieron servir como repertorio del cuento colombiano de la época.

\section{De la "versión" a la "traducción": estatuto de la traducción y del traductor en $E l$ Gráfico}

Para responder a la primera pregunta debo remitirme a la mención de un hecho: a partir de la segunda etapa de la revista desaparece la sección "Versiones de El Gráfico", en la que se publicaban los cuentos de autores de otros países, y pasa a llamarse "Cuentos Extranjeros". ${ }^{4}$ Por razones que se desconocen, desde este momento será escasa — por no decir nula - la indicación de la responsabilidad de la traducción que se presenta, pero en los

4 En la primera etapa de la revista, quien figura más asiduamente como traductor de los cuentos extranjeros es Luis Alberto Sarmiento (director y jefe de redacción de la revista en su primera etapa), con trece entradas en la base de datos de esta investigación; sus traducciones son de autores provenientes de países de (en orden de cantidad) lengua francesa (entre ellas se destacan cinco traducciones de cuentos de Henri Barbusse), inglesa, rusa y sueca. La mención de responsabilidad que más se repite en esta época para las "versiones" publicadas de los cuentos extranjeros es "El Gráfico", con 63 entradas en la base de datos. El francés, el inglés y el ruso (en ese orden) son lenguas asociadas a la mayoría de los autores traducidos. 
casos en los que esta aparece (al final del cuento, después del nombre del autor), figurará como "traducción" y ya no como "versión". Los casos a los que me refiero son dos cuentos de autores franceses: uno de Mauclair (“El peñón de Sísifo”, núm. 744 de 1925) y otro de Boutet ("El señor Erigny", núm. 745 de 1925). En el cuento de Mauclair, la responsabilidad de la traducción se atribuye a un genérico "El Gráfico"; en el de Boutet, a “J.P. Gómez para El Gráfico". En ninguno de los otros cuentos revisados volverá a aparecer este tipo de menciones.

Esta situación tiene que ver con dos circunstancias: la primera, que - como mencioné al inicio de este artículo - esta segunda etapa de la vida de El Gráfico se asocia con la dinámica de una publicación periódica no especializada en literatura y esto implica que el público al que va dirigida es un grupo menos interesado en quién hace la traducción que en su contenido-significado (la historia); la segunda circunstancia es que la noción de "versión” se asociaba más, en la época (sobre todo, a partir del modernismo), con un tipo de traducción hecha por autores literarios reconocidos $-\mathrm{y}$, por lo general, dirigida a sus pares-, quienes usaban el ejercicio de traducción como una extensión del ejercicio creativo.

En la "versión", la traducción se proponía a modo de adaptación del texto original, en la que el traductor era libre de interpretar el estilo y la intención del autor traducido, más con el propósito de nutrir el propio (Aparicio, 1991). Así, se aceptaba que la traducción presentaba "diferencias considerables con respecto ya sea al texto de partida o a otras traducciones de la misma obra" (Bastin, Echeverri y Campo, 2004, p. 75). De ahí que en las publicaciones periódicas colombianas de la época con mayor prestigio literario y especialización se imprimieran diferentes traducciones (versiones) de un mismo poema, el tipo de texto más traducido en la época, en Colombia (Bedoya Sánchez, 2012; García Maffla y Sierra Mejía, 1999).

En El Gráfico, el paso de versión a traducción tendría que ver, entonces, con una práctica en la que lo importante es que el lector comprenda, en sus líneas generales, el sentido del texto de partida. Este lector, a diferencia del de las publicaciones periódicas literarias especializadas (perteneciente, en su gran mayoría, al grupo de los intelectuales o de los aspirantes a tales), no tenía autoridad para evaluar los textos en su idioma original o las diferentes versiones que se hicieran de estos.

Esta transformación en la historia de la traducción en Colombia nos enfoca en la diversificación de los modos de esa traducción, ya no solo realizada por autores-traductores autoritas, sino también por traductores "generalistas", quienes trabajan por encargo y que, a diferencia de los autoritas, no son susceptibles de reivindicar una imagen pública o una función-autor (Zapata, 2015). Este último tipo de traductores surgió de manera más visible cuando aumentaron las publicaciones periódicas dirigidas a un público lector menos especializado-letrado.

La poesía, a diferencia del cuento, era un género que, por su alto grado de legitimación en el campo intelectual colombiano, hacía que su traducción reprodujera esta imagen de autor-creador para quien la firmaba, sobre todo si aparecía en una publicación literaria especializada. El cuento, por ser un género aún marginal dentro del sistema literario colombiano del momento, no permitía que el estatuto del traductor accediera al estatuto de autor, es decir, sus traductores eran, igualmente, marginales dentro de esta práctica, mucho más si sus traducciones aparecían en publicaciones periódicas no especializadas.

Los traductores generalistas-anónimos no son un caso excepcional en Colombia; la mayoría de las publicaciones periódicas de la época (españolas y argentinas, según los casos que se consultaron para esta investigación), dirigidas más al público "general", tampoco indicaban el nombre del traductor o la fuente de sus traducciones (Ezama Gil, 1992; Willson, 2011). Todo parece indicar que, solo en pocos casos, la dirección de estas publicaciones contrataba una traducción 
original para los textos en otro idioma que deseaba divulgar y, la mayoría de las veces, las traducciones provenían de otras publicaciones periódicas, sin que pareciera necesario mencionar la fuente. ${ }^{5}$ Esta práctica común puede explicarse, por un lado, porque, en la época, las traducciones eran mal pagadas y quienes se dedicaban a ellas debían hacerlas en cantidades "industriales" (Wilfert, 2002), en desmedro de su calidad; poco podía interesarle, entonces, al traductor, que su nombre apareciera asociado a un producto que difícilmente podría darle prestigio. ${ }^{6}$

Por otro lado, la falta de indicación de la fuente de la traducción podría entenderse a partir del marco legal del derecho de autor que se tenía en la época en el país, respecto a las traducciones. En 1933, el Gobierno aún no había suscrito convenios para la protección de los derechos de los autores extranjeros en Colombia;

[...] de consiguiente [era posible] hacer la traducción de la obra [...], sin más limitaciones que las prescritas en el inciso del Art. 39 de la Ley 32 de 1886 [indicar el nombre del autor del texto de partida] ("Carta del director de Educación Pública de Medellín”, 1933).

Ni los derechos de los autores extranjeros y menos los de sus posibles traductores (extranjeros también, presumiblemente) eran reconocidos en Colombia. Esta situación solo empezará a cambiar en 1939, cuando el Gobierno adhirió los acuerdos de la "Convención sobre propiedad literaria y artística”, suscritos en la IV Conferencia Internacional

5 Como se puede inferir, por ejemplo, de la traducción de un cuento ruso publicado en El Gráfico ("La amada ciudad de Kalitin", de Averchenko, núm. 785 de 1926), en el que es visible el uso de la variante del castellano español - a menos que se haya encargado la traducción a un español residente en Colombia-.

6 También podría explicarse de la siguiente manera: es posible que el traductor tuviera la calidad de autoritas en su sistema literario de partida (donde se hizo o se publicó la traducción originalmente), pero al pasar al sistema literario colombiano a través de El Gráfico, perdiera esta condición y pasara a ser "generalista", pues al ser desconocido en Colombia, poco importaba conservar y obtener prestigio de su imagen de autor.
Americana, realizada en Buenos Aires, en 1910, y en la cual figuraban acuerdos específicos con Estados Unidos, Francia y España (Carpeta 4, 1939). En este sentido, los directores de El Gráfico no incurrían, pues, en ninguna falta al no citar la fuente de sus traducciones, en los casos en los que estas provinieran del extranjero. El momento de cierre de El Gráfico coincidiría, así, con una fase en la historia de la traducción en el país que marcaría el paso hacia un reconocimiento mayor del estatuto autorial de los traductores.

Desde otra perspectiva, la reproducción de textos ya traducidos al español demostraría la creciente oferta y demanda de impresos dirigidos al público hispanoamericano. Si bien en el siglo XIX este ya era un mercado bastante considerable, el aumento de los índices de alfabetización y el inicio de la masificación de las ciudades, a principios del siglo $\mathrm{xx}$, produjeron la ampliación de un público lector que ya no tenía que aprender francés o inglés para entender las ediciones que llegaban del exterior, sino que recibía esas publicaciones en su idioma; aunque para la mayoría, que no podía comprar los impresos en su idioma original, estos textos extranjeros traducidos llegaban mediados doblemente: por las publicaciones extranjeras que los traducían y por las publicaciones periódicas nacionales que los reproducían. En este punto, el análisis de la práctica de la traducción en El Gráfico se entronca con el estudio de "la circulación de textos de manera que refleja el sistema económico mundial" (Ferrari, 2012, p. 22).

¿Qué publicaciones extranjeras llegaban a Bogotá en las primeras décadas del siglo Xx? Bien sabemos que, en Colombia, aún durante las primeras décadas del siglo xx, el libro era, en su mayoría, un bien importado de Europa y que este provenía, en mayor cantidad, del comercio de impresos francés y español (Gómez Mendoza, 2008). ${ }^{7}$ La aparición, en El Gráfico, de una gran mayoría de textos provenientes de autores franceses, seguida de la

7 Esto se infiere, además, por las pocas referencias a publicaciones y a autores latinoamericanos que figuran en $\mathrm{El}$ Gráfico. 
de autores españoles y rusos no tendría que ver, entonces, solamente con una selección de la dirección de la revista (con su ethos), sino, sobre todo, con una selección ya mediada por el mercado de impresos francés y español (en ese orden), es decir, por lo que, desde su perspectiva, se debía traducir y enviar hacia Hispanoamérica. Francia no solo era un sistema cultural con una acumulación notable de capital simbólico para Colombia (y para el mundo occidental) desde el siglo XIX, sino también con un poder económico para desplegar ese capital, a través del control del comercio de impresos (libros y publicaciones periódicas, sobre todo, pues estas ofrecían mayor diversidad de títulos y de contenidos), que tuvo desde inicios del siglo XIX en Latinoamérica y, específicamente, en Colombia (Murillo Sandoval, 2016). El poder simbólico y económico de Francia y, en particular, de París, se ve reflejado en dos hechos: el primero, que la literatura rusa que llegó a Colombia en la época fue mediada asimismo, muy posiblemente, por las publicaciones francesas; el segundo, que muchos autores españoles e hispanoamericanos - como otros muchos del mundo, incluida una buena cantidad de colombianos- se dieron cita en París y desde allí tradujeron y compilaron gran cantidad de textos que luego llegaron a España e Hispanoamérica, ${ }^{8}$ así como también crearon publicaciones periódicas (en francés y en español) para visibilizar la literatura latinoamericana. ${ }^{9}$

Sobre el primer hecho, Blaise Wilfert (2002) explica cómo, hacia finales del siglo XIX, los simbolistas franceses (entre ellos, André Gide, Francis de Miomandre, Remy de Gourmont, Valery Larbaud) y los traductores se encargaron de difundir la

8 Entre ellos, dos libros impresos por la poderosa editorial Garnier Hermanos (París): Cuentos escogidos de los mejores autores franceses contemporáneos, de 1893, traducidos al español por Enrique Gómez Carrillo, y Cuentos escogidos de los mejores autores castellanos contemporáneos, de 1894, compilados también por Gómez Carrillo.

9 Entre ellas, La Revista de América y la Revue de L'Amerique Latine, ambas de las dos primeras décadas del siglo $\mathrm{xx}$. literatura rusa y la nueva novela inglesa en las publicaciones parisinas, con el objetivo de subvertir las normas literarias y de "liberar" la lengua francesa. Hacia 1885, en cambio, fue creciente la hostilidad, entre los escritores, hacia la literatura extranjera; es en ese momento cuando autores como Charles Maurras, Maurice Barrès y Paul Bourget, junto con algunos simbolistas que cambian de parecer estético, se convierten en defensores de un nacionalismo literario (Wilfert, 2002). Pese a este nacionalismo, Carmen Pujante Segura (2012) ha demostrado cómo, aún en la primera mitad del siglo XX, en las publicaciones periódicas francesas, los autores ingleses, rusos y españoles (en ese orden) eran los extranjeros más traducidos en sus páginas. Por su parte, Baldomero Sanín Cano, crítico literario colombiano, afirmaba en un ya famoso artículo de 1888, publicado en La Sanción: "El lector debe estar convencido de que aquí traducimos del francés, lo mismo a Byron y a Goethe que a Petofi y a Pushkin" (1987, p. 28).

Estas dos situaciones me llevan a una conclusión parcial acerca de la muy probable mediación francesa en la recepción de la literatura rusa en Colombia: por un lado, la presencia de Chéjov y de Averchenko en El Gráfico puede deberse a este interés de los simbolistas franceses por la difusión de la literatura rusa; sin embargo, la revista colombiana no dejó de divulgarlos hasta su cierre y, en cambio, no publicó cuentos de Barrès ni de Maurras, y de Bourget solo publicó uno, en 1933. Chéjov y Averchenko se habían consagrado antes de la Revolución de Octubre (1917) y Averchenko tuvo que exiliarse por sus constantes críticas al régimen comunista de su país. La presencia de estos dos autores en El Gráfico, entre 1920 y 1940, muy seguramente se debió a su consagración como maestros del género cuento y a su ya muy continua presencia en las publicaciones francesas dirigidas a España e Hispanoamérica, pasando por alto los debates sobre el nacionalismo francés. Por otro lado, las palabras de Sanín Cano confirmarían que las traducciones hechas en Colombia de autores provenientes de otras 
lenguas (inglesa, alemana, húngara y rusa, según los casos que refiere el crítico) se hacían a partir de traducciones secundarias provenientes del francés, al menos, para finales del siglo XIX.

Un dato que puede dar mejores luces acerca de este aspecto de la práctica de la traducción en $E l$ Gráfico es la referencia que hay allí a publicaciones periódicas españolas y que demostraría su alta circulación en las primeras décadas del siglo xx en Colombia. Según la investigación de Ángeles Ezama Gil (1992) para el cuento español de entre siglos publicado en la prensa, y la de Pujante Segura (2012) para el de la primera mitad del siglo $\mathrm{xx}$, los cuentos de autores franceses también fueron los extranjeros más traducidos-publicados (a los que les siguieron - a distancia- los rusos y los ingleses, ambos con porcentajes similares) (Ezama Gil, 1992). Así, se infiere que, tanto desde las publicaciones (libros y prensa) provenientes de Francia y de España, el sistema literario francés fue, todavía para las primeras décadas del siglo $\mathrm{xx}$, una mediación ineludible para el hispanoamericano, ya fuera de manera directa a través de las publicaciones francesas dirigidas al público hispanoamericano, o de las españolas. ${ }^{10}$ En consecuencia, puedo afirmar —en palabras de Susan Bassnett y Harish Trivedi-que

[...] la traducción no es una actividad inocente y transparente, sino que está altamente cargada de significación en todos los niveles; raramente, a lo mejor nunca, involucra una relación de igualdad entre textos, autores o sistemas (1999, p. 3). ${ }^{11}$

A pesar de que la perspectiva metodológica que proponen las transferencias culturales, la

10 Interesa señalar aquí, para desarrollar en próximas investigaciones, que mientras para Francia resultaban más importantes los autores ingleses sobre los rusos, para los sistemas literarios español y colombiano pareció ser al contrario.

11 La traducción es mía. La cita textual es la siguiente: "Translation is not an innocent, transparent activity but is highly charged with significance at every stage; it rarely, if ever, involves a relationship of equality between texts, authors or systems". literatura comparada (en su fase de desarrollo actual) y la literatura mundial enfatice en desdibujar las nociones hegemónicas de centro y periferia (Espagne, 2013; Thérenty, 2015), el sistema económico mundial y la diferencia entre capitales simbólicos entre un país y otro imposibilitan pasar por alto la desigualdad que continúa manteniéndose en las prácticas de traducción y en los procesos de recepción de la literatura. Para el caso preciso de la literatura latinoamericana, "la calidad de $[\mathrm{su}]$ producción cultural [...] excede su condición económica y política periférica, [...] el fallo es que $[\ldots]$ no deja de estar condicionada por ella" (Ferrari, 2012, p. 26). No obstante, en medio de estas desigualdades económicas y simbólicas que impiden la siempre tan anhelada "libre circulación" de los objetos culturales, surgen interesantes formas de apropiación de elementos de los sistemas literarios extranjeros, por parte de los países pertenecientes a sistemas literarios periféricos, que procuran desestabilizar la aparente unidireccionalidad de ciertos casos de importación literaria y que permiten concebirla, realmente, como una transferencia cultural (Bastin, Echeverri y Campo, 2004; Coutinho, 2004; Espagne, 2013; Suárez de la Torre, 2015). A estas formas de apropiación cultural me refiero a continuación en relación con El Gráfico.

\section{Transferencias culturales: de Francia para Colombia (pasando por España y Rusia)}

La metodología de las transferencias culturales (en donde se ubican de preferencia los estudios sobre las prácticas de traducción) exige el estudio de cuatro aspectos: los mediadores de la transferencia, los soportes de la transferencia, los contextos del "exportador" y del "receptor" de la transferencia, y los juegos y las estrategias que la transferencia "enmascara", es decir, sus motivaciones y sus consecuencias (Joyeux-Prunel, 2003), incluida su circulación en el contexto de llegada. Para el caso de las publicaciones periódicas en las que no aparecen el nombre del traductor ni las referencias 
bibliográficas, es decir, en las que la figura del mediador se hace “invisible”, Juan Zapata (2016) propone que el soporte material (la publicación periódica en donde se reprodujo o publicó la traducción) actuaría como vector único de la transferencia.

La revista El Gráfico, como soporte de estas transferencias culturales, sería la "escena de enunciación" (Zapata, 2016, pp. 8-9) en la que sus directores y jefes de redacción, como mediadores, presentaron un proyecto de establecimiento de la cuentística francesa, de la rusa y de la española como modelos preferibles del género. Los contextos, juegos y estrategias de esta transferencia tienen que ver con la noción de apropiación, entendida por Saglia, dentro de la práctica de la traducción, como

[...] producción de textos que no son simplemente consumidos por la lengua y la cultura de llegada, sino que, a su vez, se vuelven creativos y productivos, estimulando reflexiones, teorías y representaciones en el contexto cultural de llegada (2002, p. 96). ${ }^{12}$

En términos generales, el papel desempeñado por los cuentistas franceses, españoles y rusos en esta segunda etapa de la vida de El Gráfico, dentro del proceso de producción de prosa de ficción breve colombiana, fue lo que Itamar Even-Zohar (1999) denomina como la elaboración de un "nuevo repertorio" (por repertorio se entiende: las reglas y los materiales indispensables para elaborar y consumir cualquier producto literario). Cuando el repertorio de un sistema literario es limitado, los agentes del campo intelectual suelen acudir a otros sistemas para ampliar ese conjunto de reglas y materiales.

12 La traducción es mía. La cita textual es la siguiente: "The concept and practice of appropriation may thus reconfigure the status of translation as the production of texts that are not simply consumed by the target language and culture but which, in turn, become creative and productive, stimulating reflections, theorizations and representations within the target cultural context".
A continuación me concentro, sobre todo, en los casos de los autores franceses y de los rusos más difundidos, pues, a diferencia de estos autores, entre los españoles no hay ninguno que se sostenga en la publicación por muchos años; los tres cuentos de Gómez de la Serna, divulgados en 1939, son disímiles y a partir de ellos es muy difícil establecer una caracterización en conjunto de su repertorio.

Desde la instauración del gobierno regeneracionista (ultraconservador), en 1886 (que se extiende durante la Hegemonía Conservadora hasta 1930), los poderes oficiales buscaron reestablecer las relaciones con España y, en consecuencia, una moral apegada al catolicismo y al Partido Conservador; por su parte, quienes comercializaron bienes culturales encontraron las maneras para no ser completamente obedientes a estos objetivos. ${ }^{13}$ De hecho, sorprende leer los artículos editoriales de El Gráfico, escritos por Luis Eduardo Nieto Caballero, antes de la llegada de los gobiernos liberales (República Liberal: 1930-1946), por su profundo sentido crítico frente al gobierno conservador y su preocupación por la suerte de los liberales y del país: "La iglesia, como toda asociación poderosa, tiende a la absorción, al imperialismo, y el liberalismo calla, en su daño se ejercerán después las grandes influencias que el

13 Una de las formas más visibles de restablecimiento de ese "protectorado" moral español fue por medio del aumento de las relaciones comerciales con la "madre patria", finalidad que el país europeo acogía con beneplácito, para no ver menguados sus intereses económicos en el continente. Para la muestra, un botón: Francia se había convertido en el mayor exportador de libros en castellano y de vinos para América Latina (Cañadas, 1920, p. 8). España necesitaba afirmar su dominio económico y cultural sobre el continente, sobre todo después de la pérdida de sus últimas colonias en América al finalizar el siglo XIX, pero sabía que solo podría hacerlo si mitigaba su "leyenda negra"; para lograr esto, los aliados políticosculturales latinoamericanos ("publicistas" de la cultura hispánica), que tenía en su territorio y al otro lado del océano, fueron fundamentales y tan efectivos que, en el caso de Colombia, las transacciones comerciales con la editorial francesa Hachette se redujeron (Murillo Sandoval, 2016). 
clero inteligente ha ido ganado"; esto lo vaticinaba Nieto Caballero en 1925 (s. d.), una década antes de que el enfrentamiento entre liberales y conservadores, azuzado por la Iglesia católica, comenzara a pasar de las palabras a los actos sangrientos en los campos colombianos.

Las leyes de censura sobre la propiedad intelectual, expedidas durante los primeros años de la Regeneración, basadas en

[...] lo nocivas que podían ser las ideas liberales francesas y el protestantismo de otras naciones para las mentes de los colombianos, [...] llevó a fortalecer [la] vigilancia sobre los libros y los periódicos que se introducían y producían en el país (Pérez Robles, 2016, p. 235). ${ }^{14}$

En 1914, además, la Ordenanza 50 del Código de Policía estableció multa y prisión por publicar o expender impresos "obscenos" (Cuervo Ramírez, 2015). Ni el celo del gobierno conservador ni sus advertencias sobre la cultura francesa fueron óbice para que El Gráfico diera preeminencia a esta cultura en las páginas de su publicación. ${ }^{15}$ De hecho, uno de los pocos autores españoles

14 Así como los comerciantes de bienes culturales, los escritores también encontraron las maneras para mantenerse al margen de los dictámenes del gobierno regeneracionista. La preferencia de muchos autores colombianos de la época por la literatura francesa es evidente, por ejemplo, en la composición de la biblioteca de León de Greiff, en la que el 71,5\% de los libros fueron impresos en Francia y la mayoría de ellos estaba en francés. Los Panidas, grupo literario al que perteneció De Greiff, eligió la literatura francesa como modelo, en oposición a la española y al tradicionalismo que estuvo asociado a ella (Cuervo Ramírez, 2015).

15 En este sentido, resulta un poco extraño para un lector de esta época entender cómo, entre 1910 y 1930, El Gráfico logró mantener esta independencia de criterio frente a las continuas constricciones del Gobierno. Sin embargo, es necesario recordar que, por una parte, siempre resulta demasiado complicado para una autoridad controlar todas las acciones y, en este caso, todas las publicaciones (y todos sus contenidos); y, por otra, que El Gráfico, en su primera etapa, siempre mantuvo secciones dedicadas a los "héroes" de la Independencia y a un tipo de historia colombiana que exaltaba los valores patrios. Quizá, al menos, en esta etapa, esas secciones contribuyeron a (cubano-español, en realidad) que cuenta con un número considerable de publicaciones de o sobre él en la revista (en su primera etapa) no responde al modelo católico y conservador promulgado por la Regeneración: Eduardo Zamacois, uno de los mayores representantes de la literatura galante o erótica en la época. Mencionar a Zamacois es importante aquí, porque su estética guarda estrecha relación con la de la mayoría de los autores franceses, cuyos argumentos girarán alrededor del adulterio femenino (en primerísimo lugar), los dramas pasionales y, en general, los escarceos amorosos entre hombres y mujeres.

Así, la presencia de Zamacois y la de los autores franceses reafirmó un espacio para elaborar este tipo de temáticas en las obras literarias colombianas, así fuera en un circuito literario no muy legitimado por las autoridades literarias de la época, pero que incluyó cuestionamientos y situaciones que empezaban a ser cada vez más cotidianas para los lectores. De esta manera, el material de la literatura galante española y francesa funcionó como “modelo transnacional” (Andries, 2011, p. 458) y amplió el repertorio tanto para los autores como para los lectores, quienes estaban cada vez más familiarizados con los temas eróticos.

La presencia mayoritaria de autores franceses y rusos en cuanto a divulgación del género cuento en El Gráfico remite también a las principales referencias extranjeras en la época para los escritores colombianos. Entre estos, pareció percibirse una apropiación de las literaturas francesa y rusa a la manera de una escisión, entendida a partir de una clara diferenciación en los contenidos y fines expresados en cada una de ellas (aprehendidas, entonces, como un conjunto homogéneo de autores y obras). Para Tomás Carrasquilla, por ejemplo, la literatura rusa había sido muy

hacer menos visibles los otros contenidos y secciones de la revista. 
[...] propagada en los últimos años y [era] tan sencilla como la vida. Todos esos eslavos — si se exceptúa a Turgueneff [sic], que es algo afrancesado- se inclinan de preferencia [...] al pueblo y sus costumbres que a la aristocracia (1906, p. 307).

Por su parte, un comentador de El Gráfico, hablando de los cuentos del colombiano Antonio César Gaitán, echa de menos

$[\ldots]$ no $[. .$.$] esa fuerza esencial y humanamente profun-$ $\mathrm{da}$, casi demasiada, que anima de un sentido poderoso la mayoría de los cuentos rusos en toda su acre intensidad, sino, más bien [...] la mediana caracterización de los personajes que por ellos discurren (Vásquez, 1926, p. 2289).

Luego, el comentador pasa a describir lo que Gaitán ha tomado de Maupassant, como maestro del género: "El procedimiento emocional y subyugante, bien que sin alcanzar la perversa y trágica emotividad del autor" (Vásquez, 1926, p. 2289). La compasión ante la condición humana, la sencillez y la fuerza de los sentimientos tratados, y la empatía por personajes del "pueblo" serían las características de la literatura rusa; mientras que la cercanía a la "aristocracia” y el efecto emocional dramático y, en ocasiones, trágico, caracterizarían la literatura francesa.

Diferencias entre el tipo de personajes y emociones tratadas, estructura narrativa y retórica sí son evidentes en los cuentos publicados de los autores rusos y de los franceses en El Gráfico: los cuentos de los rusos se enfocarán menos en la caracterización de personajes pertenecientes a una clase social adinerada (que aparece constantemente en los cuentos franceses) que en la de las clases populares y sin medios económicos; de hecho, las pocas veces que aparece esta clase adinerada en los cuentos de Chéjov y de Averchenko es para referirse a ella de manera sutilmente crítica (Chéjov) y burlesca (Averchenko). Asimismo, mientras la retórica de los cuentos franceses obedece a la exaltación del drama y del sentimentalismo amoroso, y su estructura narrativa llega a ser predecible, la retórica de los rusos se centra en otro tipo de emociones y de situaciones que producen estructuras narrativas más complejas y se perciben como motivos de indagación sobre la condición humana en toda su ambivalencia y vulnerabilidad: la pobreza, la astucia, la superstición, las diferencias entre la vida del campo y la de la ciudad, la guerra y el lugar del escritor en un régimen totalitario.

En este punto, resulta necesario anotar que el hecho de que sean los cuentos de Chéjov y de Averchenko los que se publiquen por más tiempo en El Gráfico, sería un índice de que son sus propuestas las que terminarán imponiéndose como modelo obligado del género cuento, cuando este se independice totalmente del cuadro de costumbres, el relato folclórico, la anécdota, el cuento modernista y la crónica, y se legitime más allá de su función de texto para entretener y rellenar los momentos de ocio de los lectores de las publicaciones periódicas no especializadas en literatura. La literatura erótica seguirá desarrollándose en géneros para públicos más específicos, como la novela rosa y la pornográfica, y en otros medios como la radio, el cine, y más adelante, la televisión.

Hasta aquí el análisis de los contextos y las estrategias implícitas y explícitas en la dinámica de las transferencias culturales presentes en los cuentos divulgados en El Gráfico. Para volver al asunto de los soportes y de los mediadores de las transferencias culturales, resta mencionar que, en las revistas impresas en París, fundadas por autores latinoamericanos y consultadas para esta investigación (La Revista de América y la Revue de L'Amerique Latine), las figuras de José Asunción Silva, Baldomero Sanín Cano y Guillermo Valencia aparecen con cierta frecuencia, ya sea como autores o como objeto de comentarios; igual sucederá en las revistas contemporáneas españolas Azul. Revista Hispano-Americana (Zaragoza) y América en Europa (Barcelona), que tenían incluso corresponsales en Bogotá (en el caso de $A z u l$ ) y en cuyas páginas figuraron Silva y Julio Flórez. La presencia de estos escritores colombianos en las ediciones que sobre Latinoamérica se publicaron en Francia y en España nos recuerda la importancia del denominado modernismo para lograr 
el reconocimiento de la literatura latinoamericana fuera del continente, pese a que la percepción exotista y la subvaloración de este mismo sistema literario subsistía tanto en los comentadores franceses como en los españoles (García Calderón, 1912; Ramírez Vuelvas, 2014).

Estos soportes y mediadores de la recepción de la literatura latinoamericana en España y en Francia nos recuerdan que las transferencias culturales no se dan nunca en un solo sentido, pero tampoco nunca de la misma manera. Evidentemente, en la época que cubre esta investigación, los sistemas literarios francés, español y ruso tuvieron un contacto con el sistema colombiano, históricamente constatable a partir de soportes, mediadores y adaptaciones, y desempeñaron un papel importante en la creación de un nuevo repertorio para el cuento colombiano, tanto para los autores como para los lectores: los cuentistas rusos pasaron al sistema literario colombiano, muy presumiblemente a través del filtro del sistema literario francés; los franceses pasaron al colombiano de modo directo mediante sus publicaciones en francés y en español, pero también a través del filtro de las publicaciones españolas. En este sentido, como lo propone Espagne (2013), el caso presentado aquí demuestra que una transferencia cultural no suele presentarse solo entre dos países, dos lenguas o dos áreas culturales, pues casi siempre hay un tercero implicado.

Por otro lado, es igualmente evidente que la manera en la que el sistema literario colombiano respecto al cuento se difundió y movilizó en los sistemas literarios francés, ruso y español no es aún constatable. Fue la poesía el género que mejor identificó el sistema literario colombiano en el extranjero para el momento que se ha analizado en este artículo. Lo mostrado hasta aquí ratifica, pues, que "las transformaciones y las apropiaciones inherentes a la traducción son constitutivas de la literatura latinoamericana" (Bastin, Echeverri y Campo, 2004, p. 75), y se confirman en el conflicto permanente entre cosmopolitismo y etnocentrismo - en cuyo centro está la definición de lo "nacional" - que ha existido en la historia de nuestra literatura, así como en los desequilibrios económicos y simbólicos de los intercambios entre diversos sistemas literarios.

\section{Conclusión}

La presencia de los cuentos extranjeros en $E l$ Gráfico demuestra la importancia que tuvieron, sobre todo, los sistemas literarios francés, ruso y español y, en general, el contacto del campo intelectual colombiano con los de otros países - recordemos que el 50\% de los cuentos divulgados en esta segunda etapa de El Gráfico fueron extranjeros-, dentro del sistema del cuento literario colombiano del momento. La permanencia de los cuentos de los autores rusos en El Gráfico confirma la importancia de su modelo para los cuentistas colombianos. En este sentido, resulta interesante señalar que, pese al enorme capital simbólico que tenía para Colombia el sistema literario francés, en la época, no son los grandes nombres de los autores franceses los que figuran en El Gráfico, sino autores de un subgénero poco respetado entre las autoridades literarias (la literatura galante o erótica), quienes produjeron cuentos en cantidades industriales, perfectos para las necesidades de las publicaciones periódicas de variedades que empezaban a multiplicarse en la Colombia de inicios del siglo xx.

La manera en las que los cuentos rusos y franceses se movilizaron hasta El Gráfico permite detenerse en la dinámica de la economía de los bienes simbólicos, específicamente, en su circulación. El poderoso mercado editorial francés tenía una extensa red comercial con Hispanoamérica; también la tenía el mercado editorial español, aunque en mucha menor medida. De manera presumible, concluyo que los cuentistas rusos fueron movilizados hacia las publicaciones periódicas colombianas a través de la mediación de las publicaciones impresas en Francia, y que los franceses llegaron no solo directamente por medio de sus propios impresos, sino también de los impresos españoles en los que los autores de cuentos 
franceses fueron igualmente mayoría (después de los españoles). En este contexto, la traducción en $E l$ Gráfico tuvo un estatuto generalista, en el que poco importaba el nombre del traductor o la fuente de la traducción, frente a la preponderancia de los contenidos o de la historia traducida; esas traducciones provinieron, en su mayoría, de las publicaciones extranjeras orientadas al público hispanoamericano, que fue cada vez más importante en términos económicos.

\section{Referencias}

\section{Fuentes primarias}

Cañadas, F. (1920). La expansión del libro hispano. América en Europa. Revista Internacional Iberoamericana, (6), s. d.

Carpeta 4, Caja 1. (1939). Sección Archivo Anexo 2. Fondo Ministerio de Educación Nacional. Serie Derechos de Autor. Bogotá. Archivo General de la Nación.

Carrasquilla, T. (1906). Homilía N. ${ }^{\circ}$ 2. En contestación y acatamiento al hermano Max Grillo. Alpha, (8-9), 285-314.

Carta del director de Educación Pública de Medellín al ministro de Educación Nacional (1933). Sección Archivo Anexo 2. Fondo Ministerio de Educación Nacional. Serie Derechos de Autor. Bogotá. Archivo General de la Nación.

Charla con los lectores (1925). El Gráfico, (720), s. d.

García Calderón, V. (1912). La vida de París. La Revista de América, (5), 170-175.

Nieto Caballero, L. E. (1925). Editorial. El Gráfico, (745), s. d.

Sanín Cano, B. (1987 [1888]). Núñez poeta. En El oficio de lector (pp. 16-34). Caracas: Biblioteca Ayacucho.

Vásquez, R. (1926). Un cuentista novel. Antonio César Gaitán. El Gráfico, (795), 2288-2289.

\section{Fuentes secundarias}

Andries, L. (2011). Transferencias culturales en la prensa y los impresos entre Francia y México en el siglo XIx. Bulletin Hispanique, (113-1). Recuperado de http:// bulletinhispanique.revues.org/ 1554 .

Aparicio, F. R. (1991). Versiones, interpretaciones, creaciones. Instancias de la traducción literaria en Hispanoamérica en el siglo veinte. USA: Ediciones Hispamérica.
Bassnett, S., y Trivedi, H. (1999). Introduction: Of colonies, cannibals and vernaculars. En S. Bassnett, y H. Trivedi (Eds.), Post-Colonial Translation: Theory and Practice (pp. 1-18). Londres: Routledge.

Bastin, G. L., Echeverri, Á., y Campo, Á. (2004). La traducción en América Latina: propia y apropiada. Estudios. Revista de Investigaciones Literarias y Culturales, 12(24), 69-94. Recuperado de https:// ddd.uab.cat/pub/1611/1611_a2014n8/1611_ a2014n8a7/Georges-Bastin.pdf

Bedoya Sánchez, G. A. (2012). La traducción como práctica moderna de lo literario. El caso del suplemento El Nuevo Tiempo Literario (Bogotá: 1903-1915, 1927-1929). En F. Lafarga y Luis Pegenaute (Eds.), Lengua, culturay politica en la historia de la traducción en Hispanoamérica (pp. 31-39). Vigo: Academia de Hispanismo.

Botrel, J-F. (s. f.). La poética periodística de Clarin: el ejemplo de los cuentos. Recuperado de http://www. cervantesvirtual.com/obra-visor/la-poetica-periodistica-de-clarin--el-ejemplo-de-los-cuentos/html/ dccdf6fe-2dc6-11e2-b417-000475f5bda5_4.html.

Coutinho, E. F. (2004). La literatura comparada en América Latina: sentido y función. $V o z$ y Escritura. Revista de Estudios Literarios, (14), 237-258. Recuperado de http://www.saber.ula.ve/ bitstream/123456789/25206/2/articulo14.pdf.

Cuervo Ramírez, A. (2015). Los Panidas. Una historia de la lectura en Medellin (1913-1915) (Tesis de pregrado). Departamento de Historia, Facutad de Ciencias SocialesyHumanas, Universidad de Antioquia. Recuperado de http://docplayer.es/22169512-Los-panidas-unahistoria-de-la-lectura-en-medellin-1913-1915-anycarolina-cuervo-ramirez.html.

Espagne, M. (2013). La notion de transfert culturel. Revue Sciences/Lettres, (1). Recuperado de http://rsl.revues.org/219.

Even-Zohar, I. (1999). La posición de la literatura traducida en el polisistema literario. En M. Iglesias Santos (Comp.), Teoria de los polisistemas (pp. 223-231). Madrid: Arco. Recuperado de http://www.tau. ac.il/ itamarez\%20/works/papers/trabajos/EZPosicion-Traduccion.pdf.

Ezama Gil, Á. (1992). El cuento en la prensa y otros cuentos. Aproximación al estudio del relato breve entre 1890 y 1900. Zaragoza: Universidad de Zaragoza.

Ferrari, G. de (2012). Utopías críticas: la literatura mundial según América Latina. 1616. Anuario de Literatura Comparada, 2, 15-32. Recuperado de http://revistas. usal.es/index.php/1616_Anuario_Literatura_Comp/ article/viewFile/9478/9786. 
García Maffla, J., y Sierra Mejía, R. (1999). La traducción de poesía en Colombia. En Traductores de poesía en Colombia. Antología (pp. 13-30). Bogotá: Casa de Poesía Silva.

Gómez Mendoza, S. M. (2008). Colecciones literarias e industrias editoriales, Colombia 1920-1930. La Novela Semanal y la Biblioteca Aldeana. En C. E. Acosta Peñaloza et al. (Eds.), Literatura, prácticas críticas y transformación cultural, t. 1, JALLA Bogotá (pp. 173-182). Bogotá: Pontificia Universidad JaverianaUniversidad de los Andes-Universidad Nacional de Colombia.

Joyeux-Prunel, B. (2003). Les transferts culturels. Un discours de la méthode. Hypothèses, 1(6), 149-162. Recuperado de http://www.cairn.info/revue-hypotheses-2003-1-page-149.htm.

Marín Colorado, P. A. (2016). Diversificación del público lector en Bogotá (1910-1924). Un análisis de las revistas ilustradas El Gráfico y Cromos. Historia y $\mathrm{Me}$ moria, (13), 185-214. Recuperado de http://dx.doi. org/10.19053/20275137.5204.

Murillo Sandoval, J. D. (2016). El Estado como librero. Políticas oficiales y cultura impresa en Colombia, 1821-1886. En A. Rubio (Ed.), Minúscula y plural. Cultura escrita en Colombia (pp. 271-302). Medellín: La Carreta.

Pérez Robles, S. T. (2016). Censura y persecución. La literatura y el periodismo en la Hegemonía Conservadora, 1886-1930. En A. Rubio (Ed.), Minúscula y plural. Cultura escrita en Colombia (pp. 231-250). Medellín: La Carreta.

Pujante Segura, C. M. (2012). Relatos breves publicados en revistas: otras mediaciones literarias entre España y Francia en la primera mitad del siglo xx. Tonos. Revista Electrónica de Estudios Filológicos, (22). Recuperado de https://www.um.es/tonosdigital/znum22/ secciones/estudios-25-relatos_breves_publicados_ en_revistas-_otras_mediaciones.htm.

RamírezVuelvas,C.(2014).Recepcióndelaliteraturamexicana en la prensa española, durante la transición del siglo XIX al Xx. Valenciana, 7(14), 7-29. Recuperado de http://www.scielo.org.mx/scielo.php?script=sci_ar ttext\&pid=S2007-25382014000200001.

Saglia, D. (2002). Translation and cultural appropriation: Dante, Paolo and Francesca in British Romanticism. Quaderns. Revista de Traducció, (7), 95-119.

Suárez de la Torre, L. (2015). Estudio introductorio. En L. Suárez de la Torre y M.-È. Thérenty (Eds.), Tras las buellas de Eugenio Sue: lectura y apropiación de Los misterios de París. Siglo XIX (pp. 7-24). México: Instituto Mora.

Thérenty, M.-È. (2015). Los misterios urbanos en el siglo XIX: un primer episodio de la mundialización mediática. En L. Suárez de la Torre, y M.-È. Thérenty (Eds.), Tras las huellas de Eugenio Sue: lectura y apropiación de Los misterios de París. Siglo XIX (pp.27-52). México: Instituto Mora.

Wilfert, B. (2002). Cosmopolis et l'Homme invisible. Les importateurs de littérature étrangère en France, 1885-1914. Actes de la Recherche en Sciences Sociales, 144(1), 33-46. Recuperado de http://www.persee. fr/doc/arss_0335-5322_2002_num_144_1_2806.

Willson, P. (2011). Paraísos perdidos: la traducción en $\mathrm{Ca}$ ras y Caretas (1898-1908). En A. Pagni, G. Payás y P. Willson (Coords.), Traductores y traducciones en la historia cultural de América Latina (pp. 31-44). México: UNAM.

Zapata, J. (2015). ¿Podemos hablar de una postura del traductor? Tropelias. Revista de Teoría de la Literaturay Literatura Comparada, (24), 93-99. Recuperado de https://orbi.ulg.ac.be/bitstream/2268/184335/1/ \%C2\%BFPodemos\%20hablar\%20de\%20una\%20 postura\%20del\%20traductor\%3F.pdf.

Zapata, J. (2016). Algunas consideraciones metodológicas sobre la teoría de las transferencias. Ponencia presentada en el Seminario Permanente del Grupo de Investigación Colombia: tradiciones de la palabra. Medellín. Recuperado de https://www.researchgate. net/publication/309637472_Algunas_consideraciones_metodologicas_sobre_la_teoria_de_las_transferencias_culturales.

How to reference this article: Marín C., P. A. (2018). Cuento, traducción y transferencias culturales en la revista colombiana ilustrada El Gráfico (1925-1941). Íkala, Revista de Lenguaje y Cultura, 23(3), 521-534. DOI: 10.17533/udea.ikala.v23n03a08 